\title{
SEOM clinical guidelines for cervical cancer (2019)
}

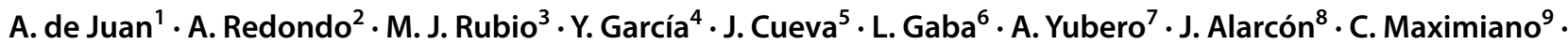 \\ A. Oaknin ${ }^{10}$
}

Received: 10 December 2019 / Accepted: 10 December 2019 / Published online: 24 January 2020

(C) The Author(s) 2020

\begin{abstract}
Cervical cancer (CC) is the fourth most common cancer in women worldwide, strongly linked to high-risk human papilloma virus infection. In high-income countries, the screening programs have dramatically decreased the incidence of CC; however, the lack of accessibility to them in developing countries makes CC an important cause of mortality. Clinical stage is the most relevant prognostic factor in CC. The new FIGO staging system published in 2018 is more accurate than the previous one since it takes into account the lymph node status. In early stages, the primary treatment is surgery—with some concerns recently raised regarding minimally invasive surgery because it might decrease survival—or radiotherapy, whereas concomitant chemo-radiotherapy is the conventional approach in locally advanced stages. For recurrent or metastatic CC, the combination of chemotherapy plus bevacizumab is the preferred therapy. Immunotherapy approach based on checkpoint inhibitors is evolving as the election therapy following failure to platinum therapy.
\end{abstract}

Keywords Cervical cancer $\cdot$ Staging $\cdot$ Treatment

A. Oaknin

aoaknin@vhio.net

A. de Juan

anade.juan@scsalud.es

A. Redondo

aredondo12@gmail.com

M. J. Rubio

mjesusrubio63@gmail.com

Y. García

ygarcia@tauli.cat

J. Cueva

jfcueva@gmail.com

L. Gaba

lydiagaba13@hotmail.com

A. Yubero

ayuberoe@salud.aragon.es

J. Alarcón

jesus.alarcon@ssib.es

C. Maximiano

conxtanza@gmail.com

1 Medical Oncology Department, H. Universitario Marqués de Valdecilla, Santander, Spain
2 Medical Oncology Department, H. Universitario La Paz, Madrid, Spain

3 Medical Oncology Department, H. Universitario Reina Sofía, Córdoba, Spain

4 Medical Oncology Department, Parc Taulí Hospital Universitari, Institut d'Investigació i Innovació Parc Taulí I3PT, Universitat Autònoma de Barcelona, Sabadell, Spain, Sabadell, Spain

5 Medical Oncology Department, Complejo Hospitalario Universitario de Santiago, Santiago, Spain

6 Medical Oncology Department, H. Clinic i Provincial de Barcelona, Barcelona, Spain

7 Medical Oncology Department, Hospital Clínico Universitario Lozano Blesa, Zaragoza, Spain

8 Medical Oncology Department, H. Universitari Son Espases, Palma de Mallorca, Spain

9 Medical Oncology Department, H. Universitario Puerta de Hierro Majadahonda, Madrid, Spain

10 Medical Oncology Department, Vall d'Hebron University Hospital, Vall d'Hebron Institute of Oncology (VHIO), Barcelona, Spain 


\section{Introduction}

$\mathrm{CC}$ represents a unique disease, due to the presence of wellknown risk factors, a very well-established etiologic agent, namely HPV infection, a long pre-invasive period which allows the use of screening tests and the availability of effective preventive vaccination [1]. In 2018, 569,847 new cases and 311,365 deaths occurred worldwide [2]. In high-income countries, the incidence of $\mathrm{CC}$ has decreased by more than a half since the introduction of screening programs. By contrast, in lower resourced settings, these rates have increased or stabilized. In Spain, about 2000 new cases of CC are expected in 2019. Squamous cell carcinoma and adenocarcinoma are the most common histological subtypes accounting for approximately $70 \%$ and $25 \%$ of all cervix cancers, respectively. HPV is a sexually transmitted DNA virus responsible for almost all CCs. More than 200 subtypes of HPV have been identified, oncogenic subtypes being only 18. Among them, HPV-16 and HPV-18 are responsible for $70 \%$ of squamous carcinomas and $90 \%$ of adenocarcinomas. Although the majority of women will clear the HPV infection during the following 2 years after the primo infection, in some of them HPV will persist, leading to the development of a pre-malignant lesion and eventually an invasive CC 15-20 years after the first infection. Vaccines against HPV have demonstrated a clear reduction in the development of pre-invasive lesions. However, the rate of implementation is variable worldwide and even within the same country. Secondary prevention has been improved with the incorporation of HPV DNA testing into the traditional Pap cytology [3]. Unfortunately, even with well-established screening programs, women could develop CC. Treatment approaches for women affected by invasive CC are presented in these guidelines.

\section{Guidelines methods}

Under the auspice of the Spanish Society of Medical Oncology (SEOM) and with the cooperation of Spanish Group of Ovarian Cancer Research (GEICO), a number of experts in the field together with two coordinators were appointed to develop this clinical practice and evidence-based guidelines. Different levels of recommendation and evidence were associated with each conclusion of the guidelines according to the US Agency for Health Research and Quality scoring.

\section{Diagnosis and staging in CC}

Early CC is often asymptomatic, while locally advanced CC (LACC) disease may cause abnormal vaginal bleeding, discharge, pelvic pain and dyspareunia. Abnormal cervical cytology or a positive high-risk HPV test should lead to colposcopy and biopsy or excisional procedures. If there is a gross palpable lesion, the diagnosis is based on biopsy. If a proper pelvic examination cannot be carried out or there is uncertainty about vaginal/parametrial involvement, it should preferably be done under anesthesia [4].

In 2018, the Gynecological Oncology Committee of FIGO updated the clinical classification. The main changes incorporated were the following: use of any imaging modality and/or pathological findings for allocating the stage; in stage IA the width of the lesion is no longer taken into consideration; stage IB now includes three subgroups as the tumor size increases $2 \mathrm{~cm}$ : stage IB1 $(<2 \mathrm{~cm})$, stage IB2 disease $(2$ to $<4 \mathrm{~cm})$, and stage IB3 $(\geq 4 \mathrm{~cm})$. The most relevant modification was the introduction of the lymph node (LN) status, indeed, the LN involvement (via histologic or radiologic assessment) was specifically designated as stage IIIC (IIIC1 pelvic LN metastasis, and IIIC2 para-aortic LN metastasis) (Table 1) [5].

Currently, imaging studies are used routinely to define more accurately the extension of disease and to allow tailoring of treatment. MRI has been shown to have the best sensitivity and specificity in assessing the size of the lesion, degree of stromal penetration, parametrial involvement and vaginal extension (Level of evidence III; Grade of recommendation B). However, the role of PET-CT with better sensitivity and specificity that MRI and CT to detect lymph nodal involvement has been progressively introduced in clinical practice as the prefered choice to stage patients [6] (Level of evidence II; Grade of recommendation B).

The role of surgical staging for diagnosing metastatic para-aortic LN is controversial. A systematic review of pretreatment assessment of the para-aortic nodes by surgery identified only one randomized study of 61 patients and concluded that there was insufficient evidence for the benefit of pre-treatment surgical staging [7]. Sentinel LN dissection in early stages has been reported with acceptable false-negative rates and good sensitivity and specificity [8].

\section{Early stages}

After careful clinical evaluation and staging, the primary treatment of early stage CC is either surgery or RT. The treatment approach is determined by the FIGO stage 2018 (Table 2) and whether fertility preservation is desired.

\section{Stage IA1}

Microinvasive cervical cancer (stage IA1) without LVSI can be managed with conisation or simple trachelectomy to preserve fertility [I, B]. Simple hysterectomy can be offered if the patient does not wish to preserve fertility. Given the low risk of LN metastasis $(<1 \%)$ in these stages, 
Table 12018 FIGO staging

\section{Stage Description}

Stage I The carcinoma is strictly confined to the cervix (extension to the uterine corpus should be disregarded)

IA Invasive carcinoma that can be diagnosed only by microscopy, with maximum depth of invasion $<5 \mathrm{~mm}^{\mathrm{a}}$

IA1 Measured stromal invasion $<3 \mathrm{~mm}$ in depth

IA2 Measured stromal invasion $\geq 3 \mathrm{~mm}$ and $<5 \mathrm{~mm}$ in depth

IB Invasive carcinoma with measured deepest invasion $\geq 5 \mathrm{~mm}$ (greater than Stage IA), lesion limited to the cervix uteri ${ }^{\mathrm{b}}$

IB1 Invasive carcinoma $\geq 5 \mathrm{~mm}$ depth of stromal invasion, and $<2 \mathrm{~cm}$ in greatest dimension

IB2 Invasive carcinoma $\geq 2 \mathrm{~cm}$ and $<4 \mathrm{~cm}$ in greatest dimension

IB3 Invasive carcinoma $\geq 4 \mathrm{~cm}$ in greatest dimension

Stage II The carcinoma invades beyond the uterus, but has not extended onto the lower third of the vagina or to the pelvic wall

IIA Involvement limited to the upper two-thirds of the vagina without parametrial involvement

IIA1 Invasive carcinoma $<4 \mathrm{~cm}$ in greatest dimension

IIA2 Invasive carcinoma $\geq 4 \mathrm{~cm}$ in greatest dimension

IIB With parametrial involvement but not up to the pelvic wall

Stage III The carcinoma involves the lower third of the vagina and/or extends to the pelvic wall and/or causes hydronephrosis or nonfunctioning kidney and/or involves pelvic and/or para-aortic $\mathrm{LN}^{\mathrm{c}}$

IIIA The carcinoma involves the lower third of the vagina, with no extension to the pelvic wall

IIIB Extension to the pelvic wall and/or hydronephrosis or nonfunctioning kidney (unless known to be due to another cause)

IIIC Involvement of pelvic and/or para-aortic LN, irrespective of tumor size and extent (with $\mathrm{r}$ and $p$ notations) ${ }^{\mathrm{c}}$

IIIC1 Pelvic lymph node metastasis only

IIIC2 Para-aortic lymph node metastasis

Stage IV The carcinoma has extended beyond the true pelvis or has involved (biopsy proven) the mucosa of the bladder or rectum. (A bullous edema, as such, does not permit a case to be allotted to Stage IV)

IVA Spread to adjacent pelvic organs

IVB Spread to distant organs

When in doubt, the lower staging should be assigned

${ }^{a}$ Imaging and pathology can be used, where available, to supplement clinical findings with respect to tumor size and extent, in all stages

${ }^{\mathrm{b}}$ The involvement of vascular/lymphatic spaces does not change the staging. The lateral extent of the lesion is no longer considered

${ }^{\mathrm{c}}$ Adding notation of $\mathrm{r}$ (imaging) and $p$ (pathology) to indicate the findings that are used to allocate the case to Stage IIIC. Example: if imaging indicates pelvic lymph node metastasis, the stage allocation would be Stage IIIC1r, and if confirmed by pathologic findings, it would be Stage IIIC1p

The type of imaging modality or pathology technique used should always be documented

Table 2 CC treatment algorithm (early stages)

\begin{tabular}{|c|c|}
\hline IA 1 & $\begin{array}{l}\text { If patient desires fertility: conization } \\
\text { If patient does not: simple hysterectomy }\end{array}$ \\
\hline IA 2 & $\begin{array}{l}\text { Hysterectomy } \pm \text { pelvic lymphadenectomy* } \pm \text { para-aortic lymphadenec- } \\
\text { tomy RT } \\
\text { If patient desires fertility: trachelectomy }+ \text { pelvic lymphadenec- } \\
\text { tomy* } \pm \text { para-aortic lymphadenectomy }\end{array}$ \\
\hline IB1 & Radical hysterectomy with pelvic* \pm para-aortic lymphadenectomy RT \\
\hline IB2 & Radical hysterectomy with pelvic \pm para-aortic lymphadenectomy RT \\
\hline IB3 & Cisplatin-based CT concurrent with external beam $\mathrm{RT}+$ vaginal $\mathrm{BT}$ \\
\hline IIA1 & Radical hysterectomy with pelvic \pm para-aortic lymphadenectomy RT \\
\hline IIA2 & Cisplatin-based CT concurrent with external beam RT + vaginal BT \\
\hline
\end{tabular}

*Sentinel lymph node dissection optional lymphadenectomy is not needed. However, in the stage IA1 with LVSI, pelvic LN dissection is recommended (Level of evidence IIB; Grade of recommendation B).

\section{Stage IA2}

Stage IA2 tumors can be treated with radical hysterectomy or radical trachelectomy (for patients who wish to preserve 
their fertility) and pelvic LN dissection (sentinel LN dissection is an option) with (or without) para-aortic LN sampling (Level of evidence IIB; Grade of recommendation B). Paraaortic node dissection is indicated for patients with known or suspected pelvic nodal disease (Level of evidence IIB; Grade of recommendation B). For patients who are not suitable for surgery or who refuse the procedure, pelvic radiation with brachytherapy (BT) is an option.

\section{Stages IB1, IB2 and IIA1}

For patients with stages IB1-IB2 or IIA1 disease, surgical approach is the preferred treatment, including radical hysterectomy type III or $\mathrm{C}$ and bilateral pelvic $\mathrm{LN}$ dissection with or without para-aortic LN sampling. Patients with IB1 stage can be treated with only pelvic lymphadenectomy (sentinel LN dissection optional). However, para-aortic node dissection is mandatory for all patients with IB2 and IIA1 stages. For selected patients with stage IB1 who desire fertility preservation, radical trachelectomy and pelvic $\mathrm{LN}$ dissection (or sentinel LN dissection) with (or without) para-aortic LN sampling is an option. In cases of patients not suitable for surgery, combining pelvic $\mathrm{RT} \pm \mathrm{CT}$ and $\mathrm{BT}$ is an alternative therapy [6]. The role of concurrent cisplatin-containing CT and RT in early stages is still under evaluation; this option has therefore to be taken with caution.

Surgical technique approach Radical hysterectomy performed using minimally invasive surgery (MIS) technique has been established as a gold standard for the last years, however, it has recently come under scrutiny.

In the phase III Laparoscopic Approach to Cervical Cancer (LACC trial) non-inferiority trial, recently published, authors reported on 631 women with FIGO 2014 stage IA1 (with LVSI), IA2, and IB1 cervical cancers, who had been randomly assigned to MIS radical hysterectomy $(n, 319)$ or open radical hysterectomy $(n, 312)$. The disease-free survival (DFS) at 4.5 years of MIS arm was $86.0 \%$ compared with $96.5 \%$ for open surgery arm (95\% CI, 21.4-24.7\%). The difference persisted after adjustment for age, body mass index, stage of disease, LVSI, lymph node involvement, and performance status score. MIS was also associated with a lower rate of overall survival (OS) (3-year rate, $93.8 \%$ vs. 99.0\%; hazard ratio for death from any cause, 6.00 ; $95 \%$ CI, 1.77-20.30). Melamed et al. using the SEER and National Cancer Databases also reported a higher mortality rate associated with MIS radical hysterectomy [9-11].

In light of the results obtained by both these studies, the ESGO Scientific Committee and Council has modified their statement, and the current ESGO recommendation regarding the approach for radical surgery for $\mathrm{CC}$ is that the open approach is the gold standard. Therefore, open surgery must be recommended as the optimal approach (Level of evidence I, Grade of recommendation A). MIS could be considered in small tumors (less than $2 \mathrm{~cm}$ ) including protective maneuvers and excluding manipulator. Risk benefit counseling must be carefully done.

Adjuvant treatment on the basis of the pathologic findings of the surgical specimen should be weighed in according to the identified risk factors. A prospective study in patients with node-negative stage IB identified intermediate risk factors for recurrence such as tumour diameter $>4 \mathrm{~cm}$, deep cervical stromal invasion and positive LVSI (Sedlis criteria). In the Gynecologic Oncology Group (GOG)-092 study, Sedlis et al. randomly assigned 227 eligible patients with at least two of three high-intermediate risk factors to adjuvant RT (50.4 Gy) versus observation. With a median follow-up of 10 years, a significant benefit has been shown for PFS HR $0.54,95 \%$ CI $0.35-0.81, p=0.007$ but not for OS (HR $0.7 ; p=0.07$ ) [12] (Level of evidence IIa; Grade of recommendation B). On the other hand, there is a group of patients which may have high-risk factors in the surgical specimens such as positive LN, positive margins and/or microscopic parametrial involvement. In this setting of patients, adjuvant CRT is indicated based on the GOG 109 study results. The GOG\#109 randomly assigned 268 women with IA2, IB and IIA stages to adjuvant RT with or without CT (cisplatin-5-fluorouracil) for four courses. The use of CT was associated with a substantially better 4 -year OS $(81 \%$ vs. $71 \%$ ) and PFS (80\% vs. $63 \%)$ and the outcomes were better for patients who completed three to four cycles of CT (Level of evidence Ib; Grade of recommendation A) [13]. Nevertheless, cisplatin monotherapy concurrent to RT is the recommended regimen, since the addition of 5-FU increases morbidity and no benefit was provided in advanced stages compared to cisplatin.

\section{Locally advanced stages}

LACC includes a heterogeneous group of tumors with different FIGO stages: IB2; II; III and IVA. In 1999, five phase III randomized trials of CRT versus RT demonstrated a reduction in the risk of recurrence by up to $50 \%$. In light of these results, in 1999 the NCI announced concurrent CRT as the new standard of care for patients diagnosed with LACC. This announcement changed clinical practice worldwide. The initial positive results were definitely demonstrated in the Cochrane individual patient meta-analysis of 13 trials [14] with an improvement in CRT of $10 \%$ and $13 \%$ in absolute 5-year OS and disease-free survival (DFS), respectively. In addition, a decreasing relative effect of CRT on OS with increasing tumor stage was observed, with absolute OS benefits of $10 \%, 7 \%$ and $3 \%$ in stages IA-IIA, IIB and III-IVA, respectively.

The most common CT regimen used concomitantly with the radiation therapy (EBRT) is weekly cisplatin at a dose of $40 \mathrm{mg} / \mathrm{m}^{2}$ (maximum $70 \mathrm{mg}$ as total dose). In patients 
not suitable for cisplatin, weekly carboplatin substitution is reasonable, although the evidence is limited. EBRT is typically administered at $1.8 \mathrm{~Gy} /$ day, five days per week for 26 days delivering a total dose of 45 to 50Gy to the whole pelvis, generally using a four-field box technique to include the uterus, cervix, adnexal structures, parametrial and pelvic LN. Patients with LACC should have treatment completed with brachytherapy (BTV) in order to deliver a high central dose to the involved cervix. There are different high-doserate dose fraction schedules employing a dose of 5.5 to 8 Gy by three to five fractions per week. Treatment delays resulting in completion of radiotherapy exceeding 9 to 10 weeks have correlated with higher rates of pelvic failure and the concurrent recommendation is to complete EBRT plus BTV within 8 weeks.

Adjuvant CT to CRT is not recommended, as there is limited evidence of benefit to justify the toxicity risks. A phase III trial compared standard of care (cisplatin concurrent to radiation) with experimental arm based on cisplatin plus gemcitabine during RT followed by two additional cycles of chemotherapy after CRT. Combination arm significantly improved PFS and OS at the expense of an increase of grade 3/4 toxicities [15]. The role of adjuvant CT merits further investigation in this high-risk population and is being addressed in an international randomized study (OUTBACK trial) (NCT01414608), that compares adjuvant CT following CRT to CRT alone in LACC patients.

In the LACC setting, neoadjuvant CT (NACT) before surgery has been largely addressed. The rationale for this approach was that NACT has the potential to eradicate micrometastases and could reduce systemic failures and may facilitate local control by surgical resection. Although metaanalysis data showed a benefit in OS (HR 0.65, 0.53-0.83), there are some caveats concerning the results: evidence is based on only a small number of trials, the comparator arm is only RT (suboptimal therapy) and patients in NACT arm also received RT after surgery.

Recently, two randomized clinical trials evaluating the role of NACT followed by surgery compared with the standard CRT in Ib2, IIA and IIB stages have been reported [16, 17] with disappointing results since none was able to show a superiority to NACT arm compared to standard arm. Therefore based on these data, concomitant CRT should continue to be the standard of care in LACC. A phase III trial (INTERLACE) (NCT01566240) that randomizes patients to NACT with weekly carboplatin and paclitaxel before CRT versus CRT alone in IB2-IVA FIGO stages is still ongoing.

Recommendations Primary cisplatin-based CRT is the treatment of choice in LACC (Lever of evidence IA; Grade of recommendation A). Weekly cisplatin during RT is the preferred choice. (Level of evidence Ia; Grade of recommendation A). Adjuvant CT after CRT is not recommended and remains to be investigated (Level of evidence Ib; Grade of recommendation $\mathrm{C}$ ). NACT before radical surgery is not a standard approach in LACC (Level of evidence Ib; Grade of recommendation A). In special settings of LACC, like no available RT or for treatment during pregnancy, NACT may be indicated (Level of evidence IIa; Grade of recommendation B).

\section{Local/regional recurrence}

Patients with suspected local or regional recurrence disease should undergo a detailed work-up before making the treatment decision. Both whole PET/CT and pelvic MRI have demonstrated high sensitivity and specificity to detect and evaluate the extension of local recurrence and distant metastases, which could have an impact on clinical management [18]. Pathological confirmation of recurrence is strongly recommended, especially in isolated and/or small lesions. Patients with limited locoregional recurrence should be considered for potentially curative treatment. Treatment options will depend on the patient performance status (PS), patient preferences, previous treatment and location of recurrence disease.

\section{Central pelvic recurrences (tumor limited to the vagina, bladder, rectum and/or parametrium)}

- Patients previously treated with surgery and/or with recurrences outside of the initial RT field: the recommended therapy will be CRT [19] (Level of evidence IIa; Grade of recommendation B). IMRT may be considered and the addition of BT could also be planned, mainly in recurrences located on the vaginal cuff.

- Patients previously treated with RT should be evaluated for pelvic exenteration (Level of evidence IIb; Grade of recommendation $\mathrm{B}$ ). This potentially curative surgery involves an extensive procedure with hysterectomy, bilateral salpingo-oophorectomy, cystectomy, and resection of anus/rectum and vulva/vagina. Depending on the location of the tumor, resection may include anterior, posterior or total pelvic exenteration [20]. Due to its high morbidity, careful patient selection is needed. Both MRI and $\mathrm{PET} / \mathrm{CT}$ are recommended as preoperative assessments to evaluate extension to the pelvic sidewall and rule out LN involvement and distant metastases. Negative surgical margin is the strongest prognostic factor associated with patient survival; therefore, this surgery should be performed in highly specialized centers.

Some selected patients with small central lesions $(<2 \mathrm{~cm})$ could be treated with radical hysterectomy instead of pelvic exenteration. RT may be considered in those patients 
in whom surgery is not indicated because of comorbidities or low probability of complete resection (Level of evidence $\mathrm{IV}$; Grade of recommendation C). Intraoperative RT is not routinely recommended. The role of neoadjuvant or adjuvant CT is not well established.

\section{Non-central pelvic recurrences}

Curative intent therapy may be considered in selected patients depending on the location, initial treatment received and PS.

- Lateral pelvic recurrences Patients previously treated with surgery will be candidates for CRT (Level of evidence IIa; Grade of recommendation B). In those patients previously treated with RT, pelvic exenterations will be reserved only for lateral pelvic recurrences that do not involve the sciatic nerve (Level of evidence IV; Grade of recommendation C).

- Localized nodal recurrences CRT or RT (external beam or stereotactic radiation) of isolated regional node recurrences could achieve long-term survival outcomes (Level of evidence IV; Grade of recommendation C).

- Oligometastatic recurrences Several local therapies (local resection, radiofrequency ablation, stereotactic RT) may result in excellent disease control with an acceptable toxicity profile in highly selected patients with isolated organ recurrences (Level of evidence IV; Grade of recommendation C).

\section{Metastatic disease}

The implementation of concurrent CRT as standard of care in LACC has dramatically decreased the risk of relapse; nevertheless, we are still witnessing recurrent disease. The risk of recurrence ranges from 16 to $30 \%$ in early stages and up to $70 \%$ in LACC. Most relapses occur within the first 2 years after diagnosis, and $50-60 \%$ of patients will have disease outside the pelvis.

Metastatic CC is usually a symptomatic and devastating situation for the patient, so palliative care has to be a priority in these patients. In patients with oligometastatic disease, mainly in the case of isolated lung metastases, surgery or stereotactic RT can be considered. For most of the patients, systemic treatment will be the main therapeutic option.

\section{First line}

Cisplatin had been for a long period of time the most active cytotoxic agent in the treatment of $\mathrm{CC}$, associated with a median OS inferior to 7 months. Because of these poor results, different ways were sought to improve them. The first approach was to increase the dose of cisplatin. The results from a GOG (Gynecologic Oncology Group) randomized phase III study established the dose of cisplatin at $50 \mathrm{mg} / \mathrm{m} 2$ every 3 weeks as a standard treatment in advanced $\mathrm{CC}$, since higher doses were associated with a greater toxicity without improvement in OS.

A remarkable change in the recurrent/metastatic CC treatment occurred with the publication of results from two important randomized clinical trials, the GOG 169 and GOG 179. Both studies compared cisplatin, single agent, to cisplatin in combination with either paclitaxel or topotecan, respectively. The combination with paclitaxel was superior in terms of RR and PFS but not in OS (8.8 vs. 9.7 months). However, the combination of cisplatin with topotecan showed statically significant improvement in all their endpoints: RR, PFS and OS. Later on, to clarify which was the most effective cisplatin doublet, the phase III trial GOG 204 was developed. Three chemotherapy regimens based on cisplatin were compared to the GOG standard of care, cisplatin/paclitaxel (CP). Even though no statically significant differences were reached, the combination of CP shows a positive trend in terms of PFS and OS, which reinforced its role as standard of care. Unfortunately, the median OS was still around 12 months [21].

On the other hand, a phase III trial of the Japanese Group (JCOG 0505) evaluated whether the replacement of cisplatin with carboplatin could make treatment more accessible to patients without compromising the efficacy. The considered reference scheme (CP) was compared to carboplatin AUC5 and paclitaxel $175 \mathrm{mg} / \mathrm{m}^{2}$. There were no differences in RR or OS. An exploratory analysis showed that in the population previously treated with cisplatin, there were no differences between the two schemes, but in those who had not previously received cisplatin, the $\mathrm{CP}$ arm was associated with better OS (median of 23 vs. $13 \mathrm{~m}$ ) [22].

The anti-angiogenic therapy in $\mathrm{CC}$ was developed due to the association between angiogenesis and invasive $\mathrm{CC}$ phenotype and prognosis. The first evidence of the role of anti-VEGF therapy in CC came from the GOG 227. In this single arm study on heavily pretreated population, bevacizumab showed promising activity warranting further investigation. The proof of concept was the phase III study, GOG 240. In the GOG 240, 452 women with recurrent, persistent or metastatic CC were randomized to 4 treatment arms: $\mathrm{CP}$ (cisplatin $50 \mathrm{mg} / \mathrm{m}^{2}$ plus paclitaxel 135 or $175 \mathrm{mg} / \mathrm{m}^{2}$ or topotecan $0.75 \mathrm{mg} / \mathrm{m}^{2} \mathrm{~d} 1-3$ plus paclitaxel $175 \mathrm{mg} / \mathrm{m}^{2}$ d1) with or without bevacizumab $15 \mathrm{mg} / \mathrm{kg}$. The primary endpoint was to determine if the addition of bevacizumab to CT would improve the OS. The study met its primary end point. The incorporation of bevacizumab significantly improved OS compared to CT alone (16.8 vs. 13.3 months; 
HR: $0.77,95 \%$ CI $0.62-0.95, p=0.007$ and PFS (8.2 vs. 5.9 months; HR $0.67,95 \%$ CI $0.54-0.82$ ). The RR was significantly higher in patients treated with bevacizumab (48\% vs. $36 \% ; p=0.008)$. In the final publication, the benefit of bevacizumab in OS was sustained by extended follow-up [23]. With a median OS of approximately 17 months, it was the first clinical trial in metastatic $\mathrm{CC}$ that showed a median OS greater than 12 months.

Regarding toxicity, it is important to mention that the use of bevacizumab was associated with the fistula development. In the final analysis of the GOG 240, the overall incidence of fistula was $15 \%$ among patients treated with bevacizumab, all of whom had had prior RT. The occurrence of clinically significant and/or severe fistula (i.e., grade 3) was 5.9\%. No fistulas resulted in surgical emergencies, sepsis, or death, and in addition to pelvic irradiation, other factors associated with fistula included pelvic disease, pre-existing hypertension, and current tobacco use. This adverse event has to be taken into consideration, and the addition of bevacizumab to $\mathrm{CT}$ regimens should be avoided in patients with rectum or bladder infiltration. Recently, results of phase II CECILIA study have been communicated, confirming the high efficacy of bevacizumab with carboplatin and paclitaxel in first line, with a rate of fistula development similar to GOG 240 [24].

\section{Summary and recommendations for the first-line treatment in advanced CC}

- The regimen of cisplatin $\left(50 \mathrm{mg} / \mathrm{m}^{2}\right)$, paclitaxel $(175 \mathrm{mg} /$ $\left.\mathrm{m}^{2}\right)$ and bevacizumab $(15 \mathrm{mg} / \mathrm{kg})$ should be considered the standard of care in advanced CC (Level of evidence I; Grade of recommendation A).

- In patients with rectum or bladder infiltration, the addition of bevacizumab should be avoided (Level of evidence I; Grade of recommendation A).

- Carboplatin (AUC5) and paclitaxel $\left(175 \mathrm{mg} / \mathrm{m}^{2}\right)$ are not inferior to $\mathrm{CP}$ for patients previously treated with cisplatin, but for naïve patients $\mathrm{CP}$ should be the preferred chemo combination (Level of evidence I; Grade of recommendation A).

- The combination of carboplatin (AUC5), paclitaxel $\left(175 \mathrm{mg} / \mathrm{m}^{2}\right)$ and bevacizumab $(15 \mathrm{mg} / \mathrm{kg})$ has shown interesting results, and could be considered for patients unfit for cisplatin (Level of evidence II; Grade of recommendation A).

\section{Second line}

To date, there is no evidence that any systemic treatment used after progression to the first line of metastatic CC improves OS compared to best supportive care. Nonetheless, for those patients who maintain good PS at the time of tumor progression, monochemotherapy (e.g., albumin-bound paclitaxel, vinorelbine, topotecan, gemcitabine, irinotecan, pemetrexed) can be recommended based on the results of several phase II trials that have shown a RR between 5 and 29\% (Level of evidence II; Grade of recommendation B). However, the duration of responses is short and PFS is less than 5 months in all these trials. In the absence of a clear significant clinical benefit of all of them, participation in clinical trials should be encouraged.

\section{Future}

Immunotherapy will probably play a very important role in the future treatment of recurrent/metastatic CC. This tumor has several factors that could predict benefit from immunotherapy, such as the presence of antigens associated with the tumor (oncoproteins E6 and E7), high mutational load, or the presence of a lymphocytic infiltrate, mainly CD4 and CD8 [25].

In a first phase Ib study, KEYNOTE-028, in which 24 pretreated patients (63\% with $\geq 2$ lines) with advanced PDL1 + CC were treated with pembrolizumab, a RR of $17 \%$ was communicated, with a median duration of response of 5.4 months and a median OS of 11 months. Subsequently, the phase II study KEYNOTE-158 was published, which included 98 patients with squamous cell CC, 79\% expressing PD-L1, treated with at least one previous line of CT, and who received treatment with pembrolizumab. The RR in PD-L1 + patients was $14.3 \%$, with a duration $\geq 6$ months in more than $90 \%$ of them [26]. These results led to the approval of this drug in US (but not in Europe) for patients with PD-L1 + CC who have progressed to a previous line of CT.

Recently, the results of activity of nivolumab monotherapy have been published. In the Phase I/II CheckMate 358 Trial [27], 19 CC patients were enrolled. Most patients had received prior systemic therapy for metastatic disease. Nivolumab showed ORR 26.3\% (95\% CI, 9.1-51.2). In addition, at a median follow-up of 19.2 months, median DOR was not reached (range, 23.3-29.5 months; + indicates a censored observation) in the five responding patients and median OS was 21.9 months (95\% CI, 15.1 to not reached). In spite of the small sample size, the results are very promising.

To determine if the checkpoint inhibitors after failure to platinum therapy would be superior to standard CT in terms of OS, the GOG 3016/ENGOT-cx9 (EMPOWER Cervical-1) was set up (NCT03257267). This randomized phase 3 trial will randomize more than 500 patients who have progressed to platinum therapy to either cemiplimab (anti-PD1) or physician choice CT.

Currently, there are several ongoing studies with immune checkpoint inhibitors, which will allow to establish more clearly the future role of immunotherapy in 
recurrent, persistent and metastatic $\mathrm{CC}$. One of the most relevant is ENGOTCx10/GEICO68-c/JGOG1084/GOG3030/ BEATcc (NCT03556839) a randomized phase III study that compares the standard treatment based on $\mathrm{CP}$ with bevacizumab to standard regimen plus Atezolizumab, in recurrent, persistent and metastatic CC in order to show an OS improvement in the experimental arm [28]. In the same line, the KEYNOTE-826 (NCT03635567) is studying the efficacy and safety of first-line treatment with pembrolizumab (MK-3475) plus CT vs. placebo plus CT in women with persistent, recurrent, or metastatic CC.

\section{Surveillance}

The recommendations of follow-up in $\mathrm{CC}$ are based on the risk of relapse of each patient, and there is no general agreement on which ones are the best. Periodic clinical history and physical examination, including vaginal, bimanual and rectal examination performed by a specialist are recommended. Low-risk patients can be assessed every 6 months for the first 2 years and high-risk patients (advanced stages or unfavorable histologies) every 3 months for the first 2 years and then every 6 months, until completing 5 years in both groups. Subsequently, annual follow-up is recommended [29] (Level of evidence III, Grade of recommendation B).

The value of cervical and/or vaginal cytology in the detection of recurrence is very limited, moreover the results may be artefacted in irradiated patients. There are no solid data in the routinely detection of HPV DNA in the follow-up of all cervical cancer patients, so it should only considered in irradiated patients.

The use of tumor markers in the follow-up is not recommended. Although the routine use of imaging tests such as CT scan or PET-CT has yet to be evaluated in a definitive manner, they are usually performed in the daily practice in either high-risk patients or if clinically indicated. (Level of evidence II, Grade of recommendation B) [30]. A reasonable follow-up schedule involves follow-up visits every $3-6$ months in the first 2 years and every $6-12$ months in years 3-5. Patients should return to annual population-based general physical and pelvic examinations after 5 years of recurrence-free follow-up [III, C].

Health education is very important in these patients, so they should recognize symptoms of a potential recurrence and also establish a healthy lifestyle, avoid becoming overweight, quit smoking, and perform regular exercise. Counceling in the use of vaginal dilators and lubricants are very important to ensure a better sex life and adequate gynecological examination. Hormone replacement therapy can be prescribed to women under 50 years old.

\section{Compliance with ethical standards}

Conflict of interest AdJ reports personal fees and other from Roche, AstraZeneca, Pharma Mar, Clovis Oncology, Tesaro, Eisai Ltd, PierreFabre and Pfizer, outside the submitted work. AR reports grants and personal fees from Pharmamar and Roche, personal fees from Lilly, Novartis, Amgen, Astra Zeneca, Tesaro and grants from Eisai, outside the submitted work. MJR has nothing to disclose. YG has nothing to disclose. JC reports Grants from Roche, outside the submitted work. LG has nothing to disclose. AY has nothing to disclose. JA reports personal fees and non-financial support from Hoffmann-La Roche and Clovis Oncology, non-financial Support from Merck Sharp and Dohme (MSD) and Pharma Mar, outside the submitted work. CM has nothing to disclose. AO reports personal fees and other from Roche, AstraZeneca, Pharma Mar, Clovis Oncology; personal fees from Tesaro, Immunogen, Genmab, Amgem, AbbVie, Merck; grants from Abbie Deutchland, Ability Pharmaceuticals, Advaxis Inc, Aeterna Zentaris, Amgen SA, Aprea Therapeutics AB, Clovis Oncology Inc., Eisai Ltd, F. Hoffmann-La Roche Ltd., Regeneron Pharmaceuticals, Immunogen Inc., Merck Sharp \& Dohme de España SA, Millennium Pharmaceutials Inc., PharmaMar, Tesaro Inc., Bristol Meyers Squibb, BMS, outside the submitted work.

Ethical standards The current study has been performed in accordance with the ethical standards laid down in the 1964 Declaration of Helsinki and its later amendments.

Informed consent For this type of retrospective study, formal consent is not required.

Open Access This article is licensed under a Creative Commons Attribution 4.0 International License, which permits use, sharing, adaptation, distribution and reproduction in any medium or format, as long as you give appropriate credit to the original author(s) and the source, provide a link to the Creative Commons licence, and indicate if changes were made. The images or other third party material in this article are included in the article's Creative Commons licence, unless indicated otherwise in a credit line to the material. If material is not included in the article's Creative Commons licence and your intended use is not permitted by statutory regulation or exceeds the permitted use, you will need to obtain permission directly from the copyright holder. To view a copy of this licence, visit http://creativecommons.org/licenses/by/4.0/.

\section{References}

1. Cohen PA, Jhingran A, Oaknin A, Denny L. Cervical cancer. Lancet (London). 2019;393(10167):169-82.

2. Bray F, Ferlay J, Soerjomataram I, Siegel RL, Torre LA, Jemal A. Global cancer statistics 2018: GLOBOCAN estimates of incidence and mortality worldwide for 36 cancers in 185 countries. CA Cancer J Clin. 2018;68:394-424.

3. Ronco G, Dillner J, Elfström KM, Tunesi S, Snijders PJ, Arbyn M. Efficacy of HPV-based screening for prevention of invasive cervical cancer: follow-up of four European randomised controlled trials. Lancet. 2014;383(9916):524-32.

4. Marth C, Landoni F, Mahner S, McCormack M, Gonzalez-Martin A, Colombo N; ESMO Guidelines Committee. Cervical cancer: ESMO Clinical practice guidelines for diagnosis, treatment and follow-up. Ann Oncol. 2018;29(Suppl 4):iv262.

5. Bhatla N, Berek JS, Cuello Fredes M, Denny LA, Grenman S, Karunaratne K, et al. Revised FIGO staging for carcinoma of the cervix uteri. Int J Gynaecol Obstet. 2019;145(1):129-35. 
6. Havrilesky LJ, Kulasingam SL, Matchar DB, Myers ER. FDGPET for management of cervical and ovarian cancer. Gynecol Oncol. 2005;97(1):183-91.

7. Brockbank E, Kokka F, Bryant A, Pomel C, Reynolds K. Pre-treatment surgical para-aortic lymph node assessment in locally advanced cervical cancer. Cochrane Database Syst Rev. 2013;28(3):CD008217.

8. Tax C, Rovers MM, De Graaf C, Zusterzeel PLM, Bekkers RLM. The sentinel node procedure in early stage cervical cancer, taking the next step; a diagnostic review. Gynecol Oncol. 2015;139:559-67.

9. Ramirez PT, Frumovitz M, Pareja R, Lopez A, Vieira M, Ribeiro $\mathrm{R}$, et al. Minimally invasive versus abdominal radical hysterectomy for cervical cancer. N Engl J Med. 2018;379(20):1895-904.

10. Melamed A, Margul DJ, Chen L, Keating NL, Del Carmen MG, Yang J, et al. Survival after minimally invasive radical hysterectomy for early-stage cervical cancer. Obstet Gynecol Surv. 2019;74(2):84-5.

11. Margul DJ, Yang J, Seagle BL, Kocherginsky M, Shahabi S. Outcomes and costs of open, robotic, and laparoscopic radical hysterectomy for stage IB1 cervical cancer. J Clin Oncol 2018;36(15_suppl):5502.

12. Rotman M, Sedlis A, Piedmonte MR, Bundy B, Lentz SS, Muderspach LI, et al. A phase III randomized trial of postoperative pelvic irradiation in Stage IB cervical carcinoma with poor prognostic features: follow-up of a gynecologic oncology group study. Int J Radiat Oncol Biol Phys. 2006;65(1):169-76.

13. Peters WA 3rd, Liu PY, Barrett RJ 2nd, Stock RJ, Monk BJ, Berek JS, et al. Concurrent chemotherapy and pelvic radiation therapy compared with pelvic radiation therapy alone as adjuvant therapy after radical surgery in high-risk early-stage cancer of the cervix. J Clin Oncol. 2000;18(8):1606-13.

14. Chemoradiotherapy for Cervical Cancer Meta-analysis Collaboration (CCCMAC). Reducing uncertainties about the effects of chemoradiotherapy for cervical cancer: individual patient data meta-analysis. Cochrane Database Syst Rev 2010;1:CD008285.

15. Dueñas-Gonzalez A, Zarba JJ, Patel F, Alcedo JC, Beslija S, Casanova L, et al. Phase III, open-label, randomized study comparing concurrent gemcitabine plus cisplatin and radiation followed by adjuvant gemcitabine and cisplatin versus concurrent cisplatin and radiation in patients with stage IIB to IVA carcinoma of the cervix. J Clin Oncol. 2011;29(13):1678-85.

16. Gupta S, Maheshwari A, Parab P, Mahantshetty U, Hawaldar R, Sastri Chopra $S$, et al. Neoadjuvant chemotherapy followed by radical surgery versus concomitant chemotherapy and radiotherapy in patients with stage IB2, IIA, or IIB squamous cervical cancer: a randomized controlled trial. J Clin Oncol. 2018;36(16):1548-55.

17. Kenter G, Greggi S, Vergote I, Katsaros D, Kobierski J, Massuger $\mathrm{L}$, et al. Results from neoadjuvant chemotherapy followed by surgery compared to chemoradiation for stage Ib2-IIb cervical cancer EORTC CGC 55994. J Clin Oncol 37, 2019 (suppl; abstr 5503).

18. Chu Y, Zheng A, Wang F, Lin W, Yang X, Han L, et al. Diagnostic value of $18 \mathrm{~F}-\mathrm{FDG}-\mathrm{PET}$ or PET-CT in recurrent cervical cancer: a systematic review and meta-analysis. Nucl Med Commun. 2014;35(2):144-50.

19. Lee YS, Kim YS, Kim JH, Ahn SD, Lee SW, Shin SS, et al. Feasibility and outcome of concurrent chemoradiotherapy for recurrent cervical carcinoma after initial surgery. Tumori. 2010;96:553-9.
20. Sardain H, Lavoue V, Redpath M, Bertheuil N, Foucher F, Levêque J. Curative pelvic exenteration for recurrent cervical carcinoma in the era of concurrent chemotherapy and radiation therapy. A systematic review. Eur J Surg Oncol 2015; 41(8): 975-85.

21. Liontos M, Kyriazoglou A, Dimitriadis I, Dimopoulos MA, Bamias A. Systemic therapy in cervical cancer: 30 years in review. Crit Rev Oncol Hematol. 2019;137:9-17.

22. Kitagawa R, Katsumata N, Shibata T, Kamura T, Kasamatsu T, Nakanishi T, et al. Paclitaxel plus carboplatin versus paclitaxel plus cisplatin in metastatic or recurrent cervical cancer: the open-label randomized phase III trial JCOG0505. J Clin Oncol. 2015;33(19):2129-35.

23. Tewari KS, Sill MW, Penson RT, Huang H, Ramondetta LM, Landrum LM, et al. Bevacizumab for advanced cervical cancer: final overall survival and adverse event analysis of a randomised, controlled, open-label, phase 3 trial (Gynecologic Oncology Group 240). Lancet. 2017;390(10103):1654-63.

24. Redondo A, Colombo N, Dreosti L, McCormack M, Nogueira Rodriguez A, Scambia G, et al. Primary results from CECILIA, a global single-arm phase 2 study evaluating bevacizumab (BEV), carboplatin $(\mathrm{C})$ and paclitaxel $(\mathrm{P})$ for advanced cervical cancer (aCC). ESMO Congress 2019, abstract \#3284.

25. Menderes G, Black J, Schwab CL, Santin AD. Immunotherapy and targeted therapy for cervical cancer: an update. Expert Rev Anticancer Ther. 2016;16(1):83-98.

26. Chung HC, Ros W, Delord JP, Perets R, Italiano A, Shapira-Frommer R, et al. Efficacy and safety of pembrolizumab in previously treated advanced cervical cancer: results from the phase II KEYNOTE-158 study. J Clin Oncol. 2019;37(17):1470-8.

27. Naumann RW, Hollebecque A, Meyer T, Devlin MJ, Oaknin A, Kerger J. Safety and efficacy of nivolumab monotherapy in recurrent or metastatic cervical, vaginal, or vulvar carcinoma: results from the phase I/II CheckMate 358 trial. J Clin Oncol. 2019;37(31):2825-34.

28. Oaknin A, Gladieff L, Colombo N, Villacampa G, Mirza MR, de Giorgi U, et al. A randomized, open label, phase III study of cisplatin and paclitaxel chemotherapy with bevacizumab (CTx plus B) with or without atezolizumab (Atz) as first-line treatment for metastatic, persistent or recurrent $(\mathrm{m} / \mathrm{r})$ carcinoma of the cérvix. J Clin Oncol 37, 2019 (suppl 15; abstr TPS5594).

29. Salani R, Khanna N, Frimer M, Bristow R4, Chen L. An update on post-treatment surveillance and diagnosis of recurrence in women with gynecologic malignancies: Society of Gynecologic Oncology (SGO) recommendations. Gynecol Oncol. 2017;146(1):3-10.

30. Elit L, Kennedy EB, Fyles A, Metser U. Follow-up for cervical cancer: a program in evidence-based care systematic review and clinical practice guideline update. Curr Oncol. 2016;23(2):109-18.

Publisher's Note Springer Nature remains neutral with regard to jurisdictional claims in published maps and institutional affiliations. 